\title{
Bicuspid aortic valves undergo excessive strain during opening: A simulation study
}

\author{
Susumu Katayama, MSc, Nobuyuki Umetani, MEnv, Toshiaki Hisada, PhD, and Seiryo Sugiura, MD, PhD
}

Objective: The objective of this study was to examine the influence of the morphologic characteristics of the bicuspid aortic valve on its disease progression by comparing the motion, stress/strain distribution, and blood flow of normal and stenotic tricuspid valves using simulation models.

\begin{abstract}
Methods: Bicuspid, stenotic tricuspid with commissural fusion or thickened leaflet, and normal aortic valves were modeled with internal blood flow. Blood flow and the motion of aortic valve leaflets were studied using fluid-structure interaction finite element analysis, and stress/strain (curvature) distributions were calculated during the cardiac cycle. To mimic disease progression, we modified the local thickness of the leaflet where the bending stress was above a threshold.
\end{abstract}

Results: Transvalvular pressure gradient was greater in the bicuspid valve compared with the stenotic tricuspid valve with a similar valvular area. The bending strain (curvature) increased in both stenotic tricuspid and bicuspid valves, but a greater increase was observed in the bicuspid valve, and this was concentrated on the midline of the fused leaflets. During disease progression analysis, severity of the stenosis increased only in the bicuspid aortic valve model in terms of valvular area and pressure gradient.

Conclusions: The characteristic morphology of the bicuspid valve creates excessive bending strain on the leaflets during ventricular ejection. Such mechanical stress may be responsible for the rapid progression of this disease. (J Thorac Cardiovasc Surg 2013;145:1570-6)

Video clip is available online.

The congenital bicuspid aortic valve (BAV) is an anomaly in which aortic cusps are fused during valvulogenesis. Resulting morphologic changes characterized by a mismatch between the total length of the free edge and the circumference of the aortic root, as well as the thickening and calcification of the leaflets, often lead to abnormal function in the valves. Although it is the most common form of congenital cardiac malformation, it affects at most $2 \%$ of the population, but the disproportionately high incidence of aortic

From the Department of Human and Engineered Environmental Studies, School of Frontier Sciences, The University of Tokyo, Tokyo, Japan.

Funding: This work was supported by the Japan Society for the Promotion of Science through its Funding Program for World-Leading Innovative R\&D on Science and Technology (FIRST Program).

Disclosures: Authors have nothing to disclose with regard to commercial support.

S.K. is currently affiliated with Mitsubishi Research Institute, Chiyoda-ku, Tokyo, Japan, and N.U. is currently affiliated with the Department of Computer Science, Graduate School of Information Science and Technology, The University of Tokyo, Tokyo, Japan.

Received for publication Feb 21, 2012; revisions received April 3, 2012; accepted for publication May 15, 2012; available ahead of print June 14, 2012.

Address for reprints: Seiryo Sugiura, MD, PhD, Department of Human and Engineered Environmental Studies, School of Frontier Sciences, The University of Tokyo, Kashiwanoha 5-1-5, Kashiwa-shi, Chiba 277-8563, Japan (E-mail: sugiura@k.u-tokyo.ac.jp).

0022-5223/\$36.00

Copyright (c) 2013 by The American Association for Thoracic Surgery doi:10.1016/j.jtcvs.2012.05.032 regurgitation or stenosis and their rapid progression have been issues in clinical cardiology. ${ }^{1-5}$

Recent studies informed by the high incidence of aortic root dilatation with BAV suggested a hereditary abnormality in the extracellular matrix as the cause of this disease. ${ }^{6}$ Meanwhile, the importance of mechanical stress as at least a modulator of disease progression is well recognized, and both experimental and simulation studies have been undertaken to identify the abnormal stress/strain state in these valves. Robicsek and colleagues, ${ }^{5}$ using high-speed videography, recorded the motion of cryopreserved bicuspid valves obtained from hemodynamically and clinically normal subjects in test circulation to find (1) excessive folding and creasing persisting throughout the cardiac cycle, (2) an extended area of leaflet contact, and (3) significant stenosis. In accordance with this report, Weinberg and Mofrad, ${ }^{7,8}$ in multiscale computer simulation studies, reported that the bicuspid valve shows greater flexure and stronger jet formation compared with the normal tricuspid valve. On the other hand, they also showed that deformation and stress cannot be directly translated to mechanical stress at the cellular level using their tissue model and thus hypothesized that mechanical stress is not a primary factor. Although further experimental examinations are needed to validate such a hypothesis, the organ-level findings should also be interpreted carefully because, in their simulation using an explicit finite element commercial code (LS-Dyna, LSTC, CA), both ventricular and aortic pressures were given as boundary conditions and only the bulk flow was analyzed. 


\section{Abbreviations and Acronyms \\ BAV $=$ bicuspid aortic valve \\ STAV-1 model $=$ stenotic tricuspid valve model 1 \\ STAV-2 model $=$ stenotic tricuspid valve model 2 \\ TAV model $=$ tricuspid valve model}

We have studied multiphysics simulations of the heart ${ }^{9-13}$ using a numeric approach for fluid-structure interaction analysis based on the arbitrary Lagrangian-Eulerian finite element method developed in our laboratory. ${ }^{14} \mathrm{We}$ also recently succeeded in simulation of aortic valves under physiologic conditions and compared their motion in aortic roots with and without the sinuses of Valsalva. ${ }^{15}$ In this study, we extended and applied our approach to the analysis of the BAV by comparing the motion, stress/strain distribution, and blood flow with normal and stenotic tricuspid valves. We also attempted in silico surgery of bicuspid valves to normalize the abnormal strain.

\section{MATERIALS AND METHODS}

\section{Modeling the Aortic Valves}

We created and compared 4 types of aortic valves with a short segment of aorta: (1) normal tricuspid valve model (TAV model), (2) stenotic tricuspid valve model 1 (STAV-1 model), (3) stenotic tricuspid valve model 2 (STAV-2 model), and (4) bicuspid valve model (BAV model) (Figure 1, $A$ ). The 2 stenotic valve models, STAV-1 and STAV-2, differed in their morphology, reflecting the respective pathologies assumed. The STAV-1 is the model of the sclerotic aortic valve often observed in elderly people. Because commissural fusion is usually absent in this form of aortic stenosis, we made the morphology of this model similar to that of a normal tricuspid valve. Instead, each leaflet was made thicker at the leaflet center according to the literature. ${ }^{16-18}$ The valve leaflets were fused at the commissure in the STAV-2 model, mimicking the changes observed in rheumatic valvular diseases. Reported morphologies of the BAV vary considerably among affected patients, but in this study, the BAV model was created by simply fusing the 2 leaflets. ${ }^{5,19,20}$ The valvular areas of the BAV and STAT-2 models at their full opening were reduced to 0.4 of the annulus area. In all models, the edge of each leaflet was made thicker according to the literature. ${ }^{17}$ As in our previous study, we introduced an anisotropic material property, originating from the fiber orientation (elastic modulus: $700 \mathrm{kPa}$ in the fiber direction; $233 \mathrm{kPa}$ in the cross-fiber direction) (Figure 1, A). ${ }^{15}$

\section{Heart and the Systemic Circulation as Boundary Conditions}

To the distal end of the models, we connected the 3-element Windkessel model of systemic circulation (Figure 1,B). The pumping action of the heart was simulated by applying a physiologic pressure wave (minimum $75 \mathrm{~mm} \mathrm{Hg}$, maximum $120 \mathrm{~mm} \mathrm{Hg}$ ) to the proximal end. In the case of stenotic valve models, we increased the peak systolic pressure so that the peak value on the distal side was kept at $120 \mathrm{~mm} \mathrm{Hg}$. This was intended to mimic the situation in which the baroreflex mechanism functions to maintain homeostasis.

\section{Disease Progression Model}

In the stenotic valve models (STAT-1, STAT-2, and BAV), we simulated the disease progression by changing the thickness of the original model depending on the magnitude of the regional strain (curvature), according to the relation shown in Figure 1, $C$. This relation is based on the following assumptions: (1) in the physiologic range of strain, the tissue structure is in equilibrium state; (2) excessive strain induces the proportional thickening of the leaflet, but its effect saturates in the range of extremely high strain. The threshold level for thickening was set at $50 \%$ of the mean strain (curvature).

\section{Computation}

The details of the strongly coupled fluid-structure interaction finite element analysis used in this study have been described. ${ }^{13-15}$ Briefly, the Navier-Stokes equation was described in arbitrary Lagrangian-Eulerian coordinates, which artificially deformed and moved according to the instantaneous deformation of the fluid-structure interface. Fluid meshes and structure meshes were generated to coincide with each other at the interface at the beginning of the analysis. Accordingly, the geometric compatibility and balance of traction forces were automatically satisfied by merging both meshes at the interface. To avoid the mesh distortion problem introduced by large movements of the aortic valve, we applied an automatic mesh reconnecting algorithm, which our group developed. Unlike conventional re-meshing algorithms, the present method does not change the nodal positions at all, and within a limited polygonal volume defined by vertices a new tetrahedral mesh division is searched so that element distortions are minimized. This algorithm is implemented in the iteration loop to establish the equilibrium of the whole system. Because mapping of the nodal values can be avoided, the deterioration of the solution has not been observed in our experience. All of the program codes were written in the laboratory.

\section{RESULTS}

\section{Severity of The Stenosis}

Figure 2 shows the valve motion and area of the 4 models at the full opening, with flow velocity profiles (Videos 1-4). Compared with the TAV and STAV-1 models (Figure 2, A and $B$ ), showing slow (thin arrows) and widely distributed flow profiles, the flow profiles in the STAV-2 and BAV models (Figure 2, $C$ and $D$ ) were concentrated in the center, and velocities were high (thick arrows). Furthermore, the streamlines in the BAV model deviated from the centerline, and a vortex behind the fused leaflets can be clearly seen. To quantitatively compare the severity of the stenosis, the left ventricular and aortic (downstream) pressures are shown below each model. As stated in the "Materials and Methods" section, the left ventricular pressure was adjusted to maintain the peak aortic pressure at $120 \mathrm{~mm} \mathrm{Hg}$. Although the valvular areas at their full opening were similar in both the BAV and the STAV-2 models, the pressure gradient was greater in the BAV model. On the other hand, in STAV-1, we changed the thickness of the sclerotic region of the leaflet (belly) by a factor of $2(0.4-0.8 \mathrm{~mm})$, but this did not create a significant stenosis in terms of morphology or hemodynamics.

\section{Strain in the Leaflets}

To gain an insight into the mechanism leading to the progression of valvular lesions, we compared the bending strain (curvature) in the leaflets of the models (Figure 3). Because the anisotropic material property of the valvular 

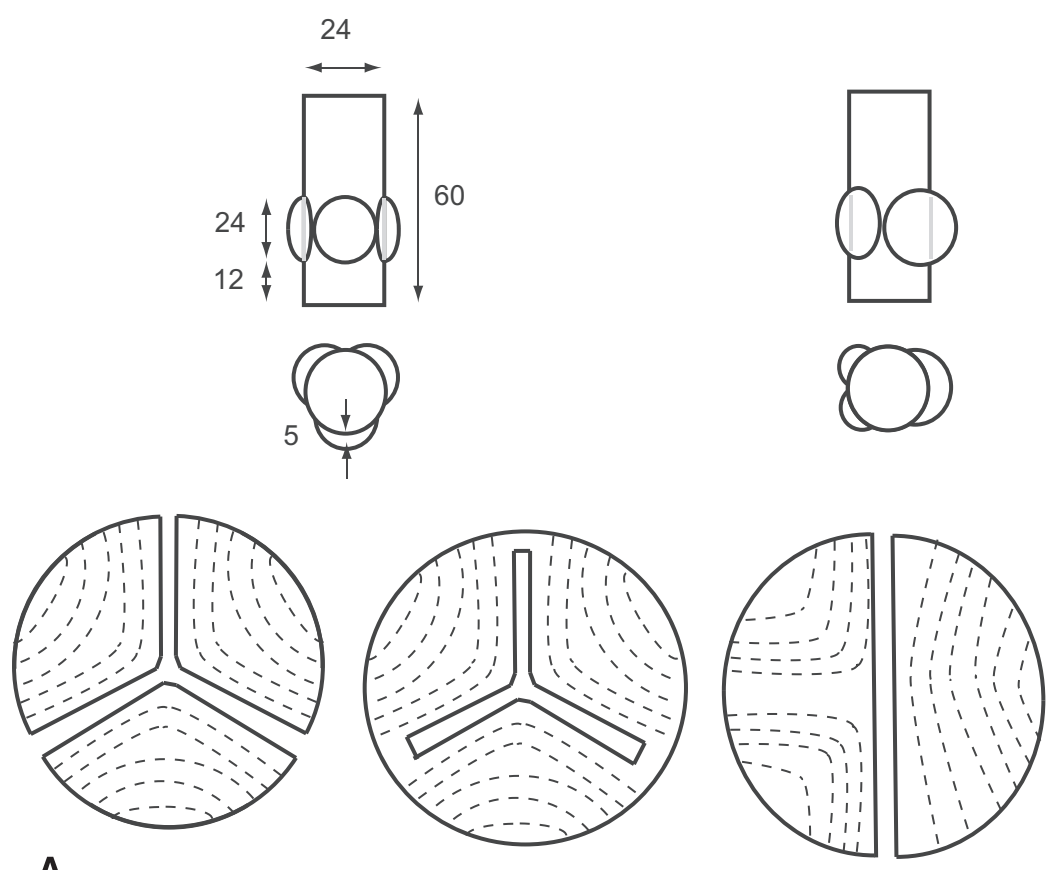

A

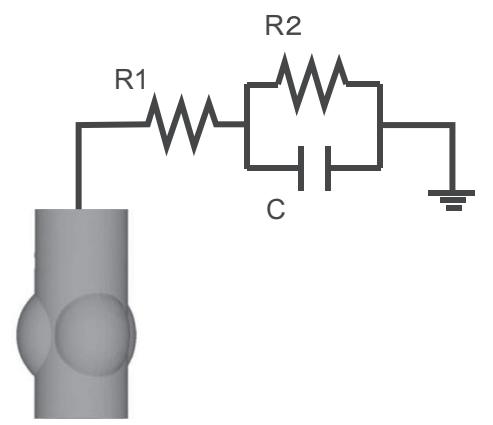

B

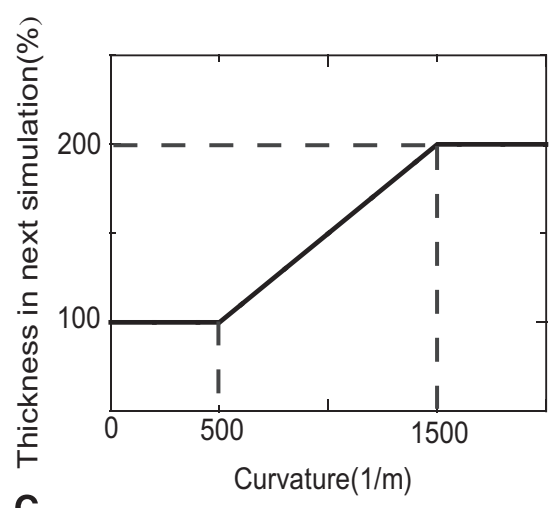

FIGURE 1. Simulation model of the aortic valve. A, Upper left: morphology of the aorta used for the TAV, STAV-1, and STAV-2 models. Upper right: morphology of the BAV model. Lower: Leaflets of the TAV and STAV-1 (left), STAV-2 (middle), and BAV (right). B, Windkessel model representing the systemic circulation. C, Relationship between the regional strain (curvature) of the leaflet and the thickness assumed for the disease progression model.

leaflet reflecting the fiber orientation was introduced in these models, we calculated the peak curvature parallel (11) and perpendicular (tt) to the fiber orientation (Figure 3, A). The curvature in both directions increased in stenotic valves (STAV-2 and BAV), but larger increases were observed in the BAV model. A decrease in curvature observed in the STAT-1 model is an indication of the increased rigidity of the leaflets.

The distributions of the curvature at the full opening phase are shown for both directions (Figure 3,B). Significant strain was observed in the STAV-1 and BAV models, and a close examination revealed that in BAV, the strain was concentrated in the midline of the fused leaflets (assumed raphe).

\section{Disease Progression Models}

It has been shown that pathologic changes in the leaflet are mainly observed in the area of greatest stress. ${ }^{16} \mathrm{We}$ simulated such pathologic processes by altering the regional thickness depending on the bending strain $(\mathrm{tt})$ distribution in the 3 stenotic valve models. We assumed that the regional thickness increases in proportion to the curvature above a threshold and reaches a plateau at high curvature (Figure 1,C), and the same relation was applied to all of the stenotic valve models. As shown in Figure 4, A, however, a significant increase in the severity of stenosis was observed only in the BAV model. In the new BAV model, the raphe moved as a rigid bar and further prevented the smooth opening of the valve, thus 
TAV

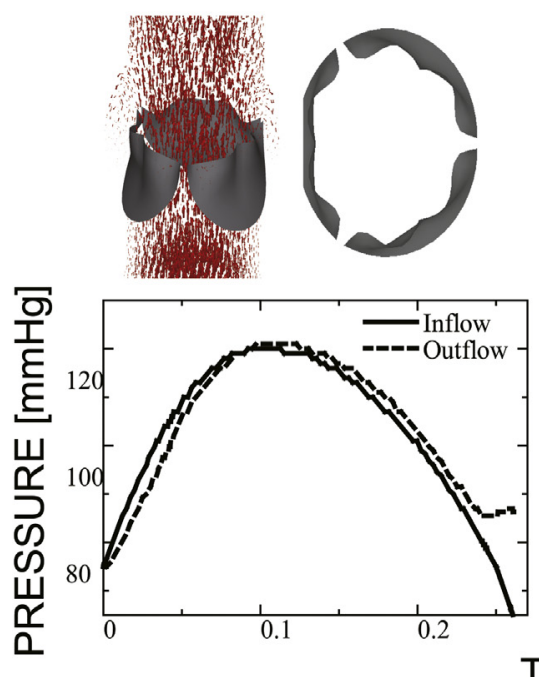

STAV-1
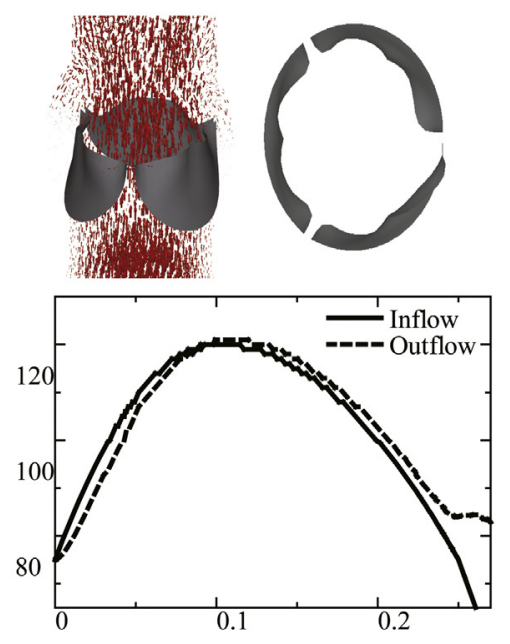

TIME [s]

\section{STAV-2}

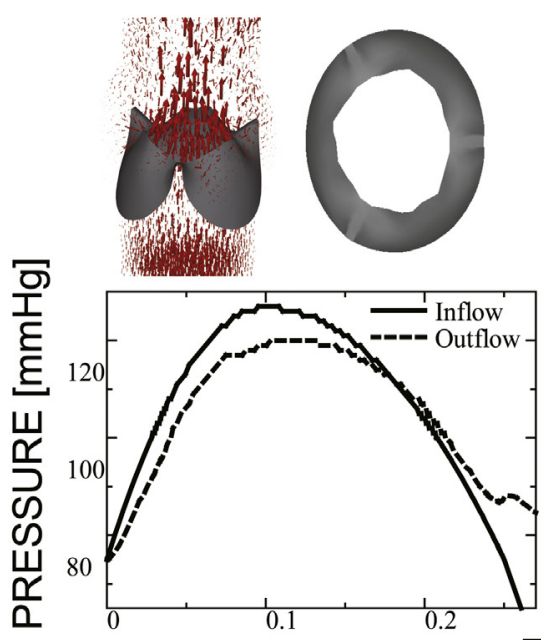

BAV

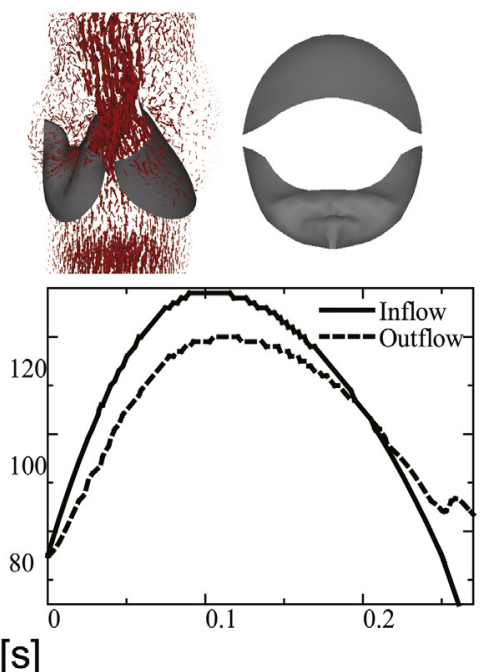

FIGURE 2. Severity of the stenosis maximal opening (upper right), flow distribution (upper left), and transvalvular pressure gradient (lower) is shown for each model. Valve opening and flow distribution were sampled at 0.1 -second intervals from the onset of ejection. Thickness of the arrow in the flow distributions indicates the flow velocity. Videos 1 to 4 correspond to this figure. TAV, Tricuspid valve; STAV-1, stenotic tricuspid valve model 1; STAV-2, stenotic tricuspid valve model $2 ; B A V$, bicuspid aortic valve.

showing a clear contrast to STAV-1, whose motion was less affected. The pressure gradient was significantly increased in BAV (7.0 vs $9.0 \mathrm{~mm} \mathrm{Hg}$ ), thus reproducing the rapid progression of the BAV stenosis. Figure $4, B$, compares the flow distribution in the axial plane between the BAV (left) and the STAV-1 models. In contrast with the symmetric flow pattern observed in the STAV-1 model, the streamline was tilted toward the rigid leaflet, and the flow collided with the leaflet from below, almost at a right angle (Video 5).

\section{Surgical Repair of Bicuspid Aortic Valve in Silico}

Although the frequent coexistence of regurgitation has made valve replacement the current first-line treatment of $\mathrm{BAV}$, plasty of the affected valve has been proposed. ${ }^{21} \mathrm{We}$ examined the efficacy of such a strategy by simulating the procedure. In this procedure, we first cut the raphe to relieve the high stress observed in this region. Howver, because this procedure introduced a flail valve during the diastolic phase, we then sutured the separated leaflet in the middle (Figure 5, A). As shown in Figure 5, B, valvuloplasty 


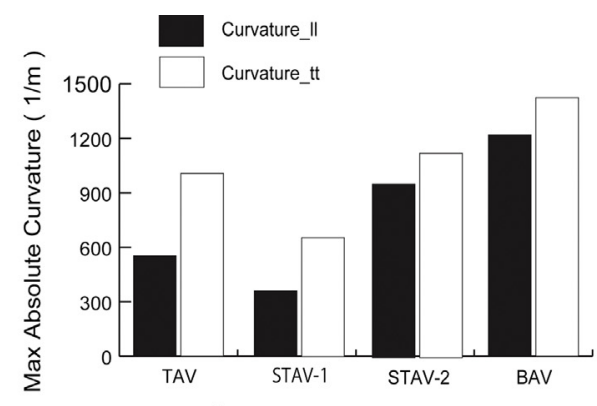

A II
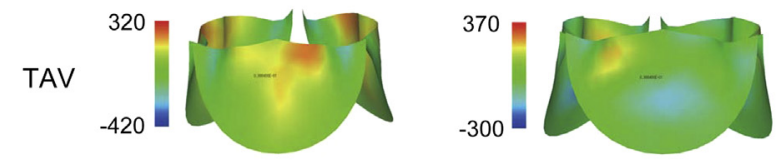

STATV-1
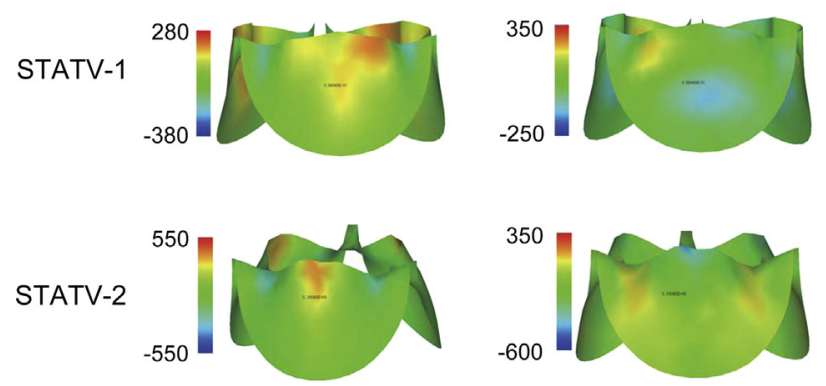

\section{B}
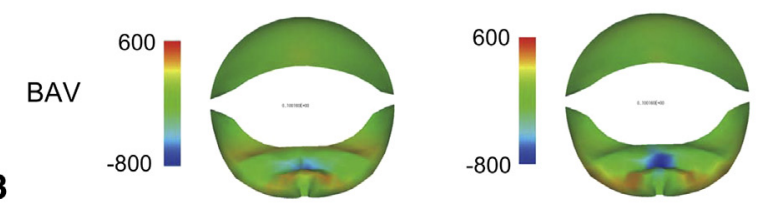

FIGURE 3. Strain (curvature) in the valve leaflet. A, Maximal curvatures in parallel (1l) and perpendicular ( $\mathrm{tt}$ ) to the fiber orientation are compared for each model. B, Distributions of the curvature (ll and $\mathrm{tt}$ ) are shown for each model. In BAV, a high strain curvature is concentrated in the commissure (raphe) of the fused leaflet. A different scale was applied to each color map to clearly show the regional variation. TAV, Tricuspid valve; STAV-1, stenotic tricuspid valve model $1 ; S T A V-2$, stenotic tricuspid valve model 2; $B A V$, bicuspid aortic valve.

recovered a wide and straight bloodstream in the orifice, and the flow converged smoothly along the widely opened leaflets (Video 6). We also found that the abnormal stress concentrated in the raphe disappeared (Figure 5, C). The pressure gradient across the valve decreased significantly (Figure 5,D), but the suture of the separated leaflet ensured the competence of the valve during diastole.

\section{DISCUSSION}

In this study, using a fluid-structure interaction finite element method, which we previously applied to the analysis of the aortic valve, ${ }^{14,15}$ we addressed the question of how the characteristic morphology of the bicuspid valve

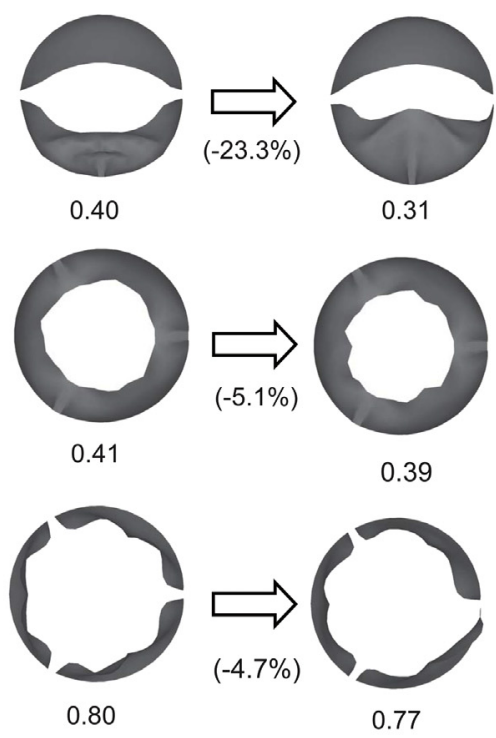

A

$$
0.80
$$

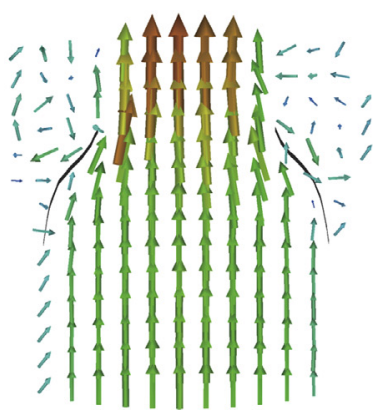

B

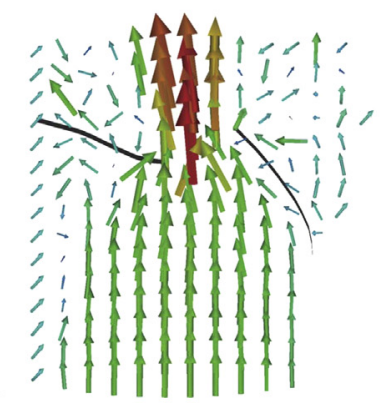

FIGURE 4. Progression of the stenosis. A, Initial thickness of the leaflets was modified according to the relation in Figure 1, $C$, in BAV (top), STAV-2 (middle), and STAV-1 (bottom). Numbers below each valve show the fraction of orifice area relative to the aortic root area. Relative reductions of the area are indicated below the arrow for each model. B, Flow distributions during the ejection are shown for BAV (left) and STAV-1 (right). Video 5 corresponds to this figure.

(ie, geometric factors) affects the hemodynamics and mechanical stress on the valve leaflets, thereby modifying the pathology of the aortic stenosis. For this purpose, we compared the bicuspid valve model with not only the normal but also the stenotic tricuspid valve models. In particular, the comparison with the 2 types of stenotic tricuspid valve models revealed interesting findings. First, although the areas of the valvular orifice of BAV and STAV-2 at their full opening were similar, the pressure gradient was larger for the BAV (Figure 2), probably because fully accelerated flow was allowed only in the central region of the ellipsoidal orifice of BAV (Figure 2). However, because we examined only one type of BAV morphology, generating a tilted flow line, we should be careful in interpreting this result, and a further analysis of models with a wide variety of valvular morphologies is needed. Also, the thickening of the leaflets center created in the STAV-1 model introduced only a minimal effect on hemodynamics, 


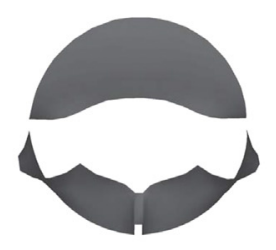

A

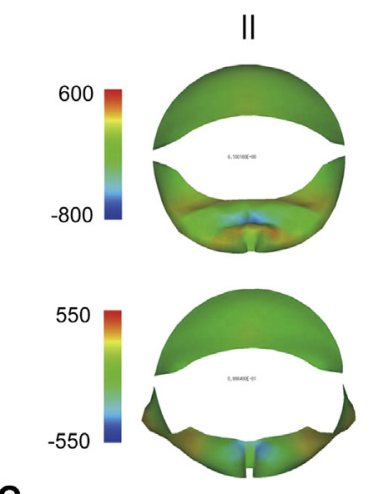

B
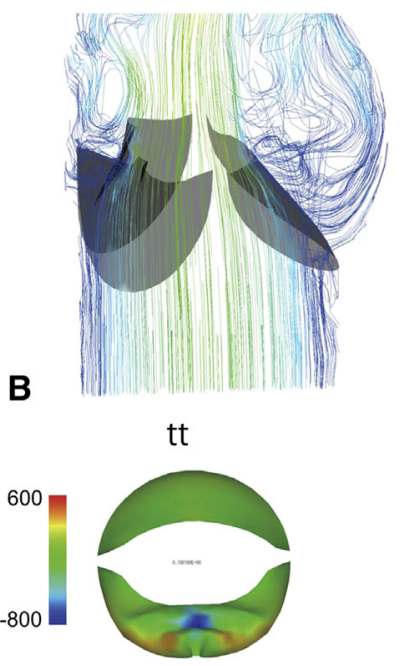

450

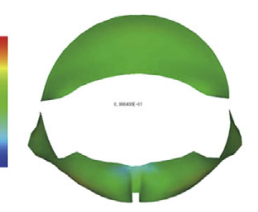

C

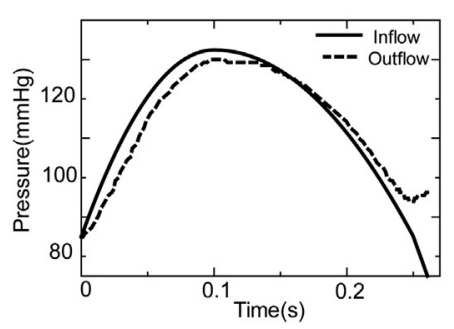

FIGURE 5. Model for surgical repair. A, Tricuspidization model. The fused leaflet was separated at the commissure and sutured the separated leaflet in the middle. B, Flow distribution after the repair. The main stream became less tilted, and the flow in the ventricular side proceeded smoothly along the leaflet. C, Curvature in parallel (11) and perpendicular (tt) to the fiber orientation after the repair. Curvature was reduced in both directions. A different scale was applied to each color map to clearly show the regional variation. D, Pressure gradient after the repair, indicating that the significant gradient had disappeared. Video 6 corresponds to this figure.

reproducing the clinical observation. ${ }^{18}$ Although the leaflets in the STAV-1 model became rigid, as evidenced by the smallest flexure among the models tested (Figure 3, A), sparing of the commissure allowed a nearly normal opening of the valve. On the other hand, despite its normal material properties, the commissural fusion in STAV-2 severely impaired the mobility of the leaflets, inducing excessive strain in directions parallel and perpendicular to the fiber orientation (Figure 3, A). In particular, a perpendicular strain was built up near the commissure. Although the histopathology was not modeled in this study, this result may also support the importance of geometric factors in valvular function and suggests the possibility of the preemptive surgery.

In addition to the converged and tilted flow of high velocity, the flow field in the BAV also showed characteristic

patterns in detail. While a rapidly rotating clockwise vortex evolved on the aortic side of the fused leaflet, the ejecting blood hit the ventricular surface almost at a right angle (Figure $4, B$ ). Such a flow pattern not only causes a high shear on the surface of the leaflet but also prevents smooth motion of the valve. Again, analyses of the different types of bicuspid valve models are needed to provide further evidence.

In the second simulation, we increased the thickness of the leaflet according to the regional strain to examine the role of mechanical factors in the progression of the disease. A significant increase in the degree of stenosis was observed only in the BAV model, and thus we could successfully reproduce clinical observations showing the rapid progression of pathophysiology in the bicuspid valve. ${ }^{3,22}$ Prominent thickening of the raphe corresponding to this simulation result has also been reported. ${ }^{6,20}$ Weinberg and Mofrad ${ }^{8,23}$ recently performed a multiscale simulation of the aortic valve to show that the macroscopic strain at the organ or tissue level is not transmitted to the cellular level to trigger the molecular signaling that leads to alterations in tissue structure. Their result seems to be in accord with a recent report identifying a genetic defect as the cause of the congenital bicuspid valve, ${ }^{6}$ but their conclusion depended solely on their assumption of a layered tissue structure, which awaits further verification. For the moment, it may be reasonable to consider the role of mechanical factors, at least as a modulator, in the progression of pathology of aortic valves.

We also simulated the valvuloplasty procedure reported in the appropriate case of the bicuspid valve. In this technique, proposed by Kawazoe and colleagues, ${ }^{21}$ fused leaflets were separated at the commissure (raphe) and the prolapse was secured by a suture placed in the middle separated leaflet margin (tricuspidization). Although the reported technique was intended for the treatment of aortic regurgitation, a similar procedure adopted in the simulation successfully reduced the pressure gradient and relieved strain in the leaflets. Although the long-term efficacy and the effect on regurgitation should be evaluated in a more patient-specific model, this result reveals the possibility of valvuloplasty as a choice for the treatment of the stenotic bicuspid valve. Moreover, stress reduction by the resection of the raphe can be applied to other techniques of aortic valve repair.

As in our previous study, ${ }^{15}$ we have shown the usefulness of the fluid-structure interaction finite element method in the analyses of aortic valve pathologies. However, we also note some limitations, which we should resolve in future studies. First, as stated above, only one form of bicuspid valve was modeled and analyzed. Considering the wide variation of BAVs, ${ }^{5,20}$ analyses with different models are needed. Such analyses may also provide a clue to the question why some BAV cases develop an insufficiency 
rather than becoming stenotic. For instance, the shift in strain distribution to the cusp attachment area may lead to the dilatation of aortic root to cause regurgitation while reducing the strain in the leaflets. Furthermore, coupling of the coronary circulation should be attempted to clarify its effect on flow in the coronary cusps. In the current axisymmetric model without coronary circulation, even the discrimination of 3 cusps was not possible. Second, for the tissue structure, we considered only fiber orientation. This is clearly an oversimplification of reality, and to fully address the question of how mechanical stress influences the tissue remodeling, ${ }^{8}$ detailed multiscale modeling would be necessary. Thickness distribution in the leaflets should also be reconsidered to reproduce the clinical situations, where variations and overlaps are observed in the histology of aortic valves affected by different causes. Finally, in this simulation, the aorta was treated as a rigid cylinder. Recent studies have identified a strong link between the phenotype of congenital bicuspid valves and a defect in the fibrilin-1 protein, which also causes dilation of the aorta, ${ }^{6,24}$ so abnormal aortic elasticity should be included in a future model.

\section{CONCLUSIONS}

We compared the function of BAVs with various types of aortic valves. The results suggest the importance of shape factors in the pathogenesis of stenotic aortic valves. A bicuspid valve model showed a larger stress at the center of valve leaflets compared with other models, which could determine the rapid progression of disease.

\section{References}

1. Roberts C. The congenitally bicuspoid aortic valve: a study of 85 autopsy cases. Am J Cardiol. 1970;26:72-83.

2. Beppu S, Suzuki S, Matsuda H, Ohmori F, Nagata S, Miyatake K. Rapidity of progression of aortic stenosis in patients with congenital bicuspid aortic valves. Am J Cardiol. 1993;71:322-7.

3. Davies MJ, Treasure T, Parker DJ. Demographic characteristics of patients undergoing aortic valve replacement for stenosis:relation to valve morphology. Heart. 1996;75:174-8.

4. Chan K-L, Ghani M, Woodend K, Burwash IG. Case-controlled study to assess risk factors for aorticstenosis in congenitally bicuspid aortic valve. Am J Cardiol. 2001;88:690-3.

5. Robicsek F, Thubrikar MJ, Cook JW, Fowler B. The congenitally bicuspid aortic valve: how does it function? Why does it fail? Ann Thorac Surg. 2004;77:177-85.
6. Fedak PWM, Verma S, David TE, Leask RL, Weisel RD, Butany J. Clinical and pathophysiological implications of a bicuspid aortic valve. Circulation. 2002; 106:900-4.

7. Weinberg EJ, Mofrad MRK. Transient, three-dimensional, multiscale simulations of the human aortic valve. Cardiovasc Eng. 2007;7:140-55.

8. Weinberg EJ, Mofrad MRK. A multiscale computational comparison of the bicuspied and tricuspid aortic valves in relation to calcific aortic stenosis. $J$ Biomech. 2008;41:3482-7.

9. Watanabe H, Hisada T, Sugiura S, Okada J, Fukunari H. Computer simulation of blood flow, left ventricular wall motion and their interrelationship by fluidstructure interaction finite element method. JSME Internatl J Series C. 2002; 45:1003-12.

10. Watanabe H, Sugano T, Sugiura S, Hisada T. Finite element analysis of ventricular wall motion and intra-ventricular blood flow in heart with myocardial infarction. JSME Internatl J Series C. 2004;47:1019-26.

11. Watanabe H, Sugiura S, Hisada T. Finite element analysis on the relationship between left ventricular pump function and fiber structure within the wall. JSME Internatl J Series C. 2003;46:1330-9.

12. Watanabe H, Sugiura S, Hisada T. The looped heart does not save energy by maintaining the momentum of blood flowing in the ventricle. Am J Physiol. 2008;294:H2191-6.

13. Watanabe H, Sugiura S, Hisada T. Multi-physics simulation of left ventricular filling dynamics using fluid-structure interaction finite element method. Biophys J. 2004;87:2074-85.

14. Zhang Q, Hisada T. Analysis of fluid-structure interaction problems with structural buckling and large domain changes by ALE finite element method. Comput Methods Appl Mech Engrg. 2001;190:6341-57.

15. Katayama S, Umetani N, Sugiura S, Hisada T. The sinus of Valsalva relieves abnormal stress on aortic valve leaflets by facilitating smooth closure. $J$ Thorac Cardiovasc Surg. 2008;136:1528-35.

16. Thubrikar MJ, Aouad J, Nolan SP. Patterns of calcific deposits in operatively excised stenotic or purely regurgitant aortic valves and their relation to mechanical stress. Am J Cardiol. 1986;58:304-8.

17. Sahasakul Y, Edwards WD, Naessens JM, Tajik AJ. Age-related changes in aortic and mitral valve thickness: implications for two dimensional echocardiography based on an autopsy study of 200 normal human hearts. Am J Cardiol. 1988; 62:424-30

18. Freeman RV, Otto CM. Spectrum of calcific aortic valve disease pathogenesis, disease progression, and treatment strategies. Circulation. 2005;111: 3316-26.

19. Edwards JE. Editorial: the congenital bicuspid aortic valve. Circulation. 1961; 23:485-8.

20. Sadee AS, Becker AE, Verheul HA, Bouma B, Hoedemaker G. Aortic valve regurgitation and the congenitally bicuspid aortic valve: a clinico-pathological correlation. Heart. 1992;67:439-41.

21. Kawazoe K, Izumoto H, Tsuboi J, Koizumi J. Tricuspidization of incompetent bicuspid aortic valve. J Thorac Cardiovasc Surg. 2003;126:908-10.

22. Campbell M. Calcific aortic stenosis and congenital bicuspid aortic valves. $\mathrm{Br}$ Heart J. 1968;30:606-16.

23. Weinberg EJ, Mofrad MRK. A finite shell element for heart mitral valve leaflet mechanics with large deformations and 3D constitutive material model. J Biomech. 2007;40:705-11.

24. Grotenhuis HB, Ottenkamp J, Westenberg JM, Bax JJ, Kroft LJ, Ross A. Reduced aortic elasticity and dilatation are associated with aortic regurgitation and left ventricular hypertrophy in non-stenotic bicuspid aortic valve patients. $J$ Am Coll Cardiol. 2007;49:1660-5 